\title{
The Extended Maxwellian View (BIGMAX): A high-intensity, high-saturation color display for clinical diagnosis and vision research
}

\author{
R. DIRK BEER, DONALD I. A. MACLEOD, and TIMOTHY P. MILLER \\ University of California at San Diego, La Jolla, California
}

\begin{abstract}
We describe a device that can display very high intensity (up to 400,000 cd/m²), high-resolution visual stimuli. The device is inexpensive, is easily controlled by a conventional computer and video card, and can be calibrated for use in vision research or clinical applications. The display is capable of presenting highly saturated, near spectral colors. Unlike Maxwellian view optical systems, our display can be viewed binocularly and does not require exacting head restraint. We describe the construction, give a design example, and describe our calibration procedure. Furthermore, we report measurements of the color gamut, spatial resolution, temporal characteristics, and the dynamic range of light intensity.
\end{abstract}

We present here a simple, relatively inexpensive display that incorporates the best features of Maxwellian view and CRT displays; it presents high-intensity, highresolution, and if desired, high color saturation images to an observer. It is suitable for use in vision research with psychophysics or brain imaging or for clinical testing. The display is constructed using a modified LCD data projector, ${ }^{1}$ a supplemental objective lens, a Fresnel lens, and a diffuser. The design is flexible; a wide range of viewing distances and angular subtenses is possible. In the configuration we built, the display presents very high intensity $\left(100-20,000 \mathrm{~cd} / \mathrm{m}^{2}\right)$, very saturated (near spectral color) images on a roughly $25 \times 25 \mathrm{~cm}$ screen positioned about $100 \mathrm{~cm}$ in front of a viewer. The images are high resolution and easily controlled with a desktop computer.

In Maxwellian view (Westheimer, 1966), a lens forms an image of a lamp filament in the pupil of the eye. Because of this, the viewed field is brightly lit; all the light from the lamp passes through the pupil. The advantage of this arrangement is a very high intensity and, if desired, highly saturated colors, if filters are used. However, Maxwellian view requires a precise alignment of the eye, which is usually achieved by having the observer bite on a dental impression. The image can be viewed only with one eye at a time (the image can be viewed with both eyes by using a more complex and difficult-to-align design). Also, stimuli are usually created using masks made from photographic lithographs, making it very difficult to present complex or dynamic stimuli.

Correspondence concerning this article should be addressed to R. D. Beer, Department of Psychology, University of California at San Diego, 5212 McGill Hall, La Jolla, CA 92093-0109 (e-mail: rdbeer@ucsd.edu).

Note-This article was accepted by the previous editor, Jonathan Vaughan.
CRT monitors have the advantage of easy control over the shape and time course of the stimuli. With a high gray level resolution video board, they can be calibrated to present precisely controlled luminance levels (Brainard, 1989). However, the maximum luminance is very low, as compared with natural light levels $\left(10^{2} \mathrm{~cd} / \mathrm{m}^{2}\right.$, as compared with $10^{4} \mathrm{~cd} / \mathrm{m}^{2}$ for a white sheet of paper in sunlight), and the color gamut is limited by the phosphors used.

Current electronic data projectors put out a lot of light $(1,000-2,500 \mathrm{~lm}$ is typical). In these projectors, a compact lamp illuminates LCD or DLP panels. These panels can be viewed in a Maxwellian configuration if that lamp, which is usually imaged near the projector's objective lens, is reimaged in the observer's pupil by a large Fresnel lens. By adding a supplementary positive lens immediately in front of the projector's objective lens (or by removing and replacing that objective lens), the LCD or DLP image can be made to coincide with and fill the Fresnel lens, a foot or two from the projector.

Such a setup yields intensities far higher than those achieved with any CRT and, in fact, higher than typically wanted. However, by introducing a holographic diffuser in the plane of the projected image, light can be uniformly dispersed over a region around the subject's eyes. This sacrifices (unneeded) intensity but (1) allows binocular observation and (2) allows a less restrictive chinrest to replace the customary dental impression. The diffuserextended Maxwellian image can even be large enough to allow binocular observation, while still providing pigmentbleaching light levels ( 5.3 log trolands are required to bleach $90 \%$ of L and M cone pigment; Rushton \& Henry, 1968; this requires a display luminance of $14,300 \mathrm{~cd} / \mathrm{m}^{2}$ with a typical 4-mm pupil). In addition, the color gamut of this display system can be greatly enlarged (approaching the physical limit of monochromatic primaries) by placing thin dichroic filters in the internal light path of three-panel projectors. Dynamic range, light level, color 
gamut, and spatial and temporal resolution are all sufficient for demanding applications in vision research.

\section{Display Optics}

Traditional Maxwellian view systems display very bright images by using a lens to focus an image of the light source (usually a lamp filament) in the pupil of the eye (Figure 1A). As long as the image of the light source is smaller than the pupil, all of the light captured by the lens enters the eye. To add a target stimulus, two lenses can be used to form the image of the light source, with a target (often a lithographic mask) placed between them (Figure 1B). The second lens, in addition to helping form the image of the light source at the pupil, has the function of forming an image of the target that ends up focused on the retina. In the traditional Maxwellian view, the second lens is placed one focal length from the target, so that rays from the target emerge parallel and the target appears at infinity to the observer. Our system uses similar principles to get light into the eye and to display a target image. The following sections will describe the optics involved in forming an image of the lamp at the observer and will separately describe the optics used to form the image of the target (LCD panels, in the case of BIGMAX).

Optical path of lamp light. We used a Proxima 9260+ projector. ${ }^{2}$ This projector contains a high-intensity discharge lamp, followed by a microlens array (Figure 2). The lens array collimates the light, which is then split into three separate pathways by dichroic color filters and mirrors. Long wavelength light illuminates the "red"

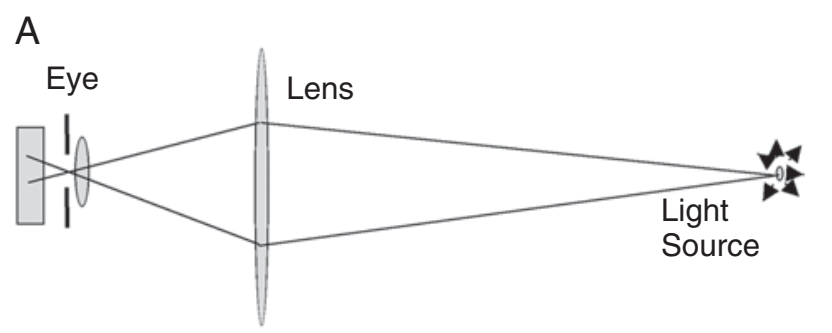

B

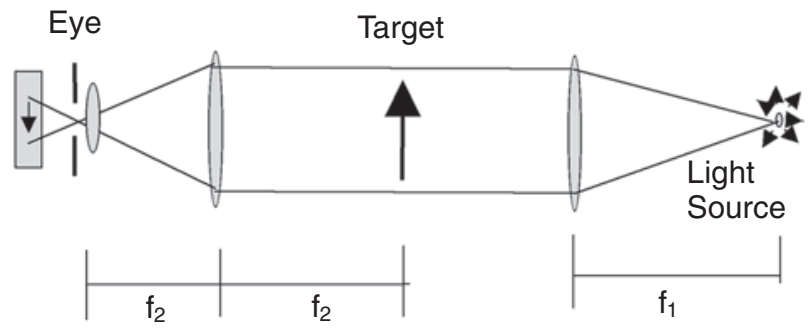

Figure 1. Traditional Maxwellian view. (A) In the Maxwellian view, a lens is placed between the light source and the eye, forming a small image of the light source at the pupil, thus allowing more light to enter the eye. (B) This panel shows how a target stimulus can be added to the Maxwellian view. Stimuli in the Maxwellian view can be much brighter than stimuli viewed normally, because a much larger proportion of the available light enters the eye.
LCD panel, medium wavelength light illuminates the "green" panel, and short wavelength light illuminates the "blue" panel. After passing through the LCD panels, light from all three pathways is recombined by a crossed set of dichroic, half-silvered mirrors and is directed out through the projector's objective lens. A small image of the lamp is formed somewhere in the objective lens to allow all light to exit the projector. This arrangement is typical of most modern LCD data projectors, as well as three-channel DLP data projectors.

In our system, a large $(27 \times 27 \mathrm{~cm}, 35-\mathrm{cm}$ focal length $)$, high-quality $(2.5$ grooves $/ \mathrm{mm})$ Fresnel lens ${ }^{3}$ is placed $50 \mathrm{~cm}$ in front of the projector and forms an image of the high-intensity lamp near the observer's pupil $100 \mathrm{~cm}$ away. Since the function of the Fresnel lens is to focus the lamp light near the observer's pupil and not to focus the viewed image, its quality and groove pitch are usually not critical. However, excessively coarse grooves on the Fresnel lens could appear as a fine pattern of luminance variation in the viewed image. At the viewing distance in our design, the grooves are not visible: There are 44 grooves/deg in the Fresnel lens - at the worst case, equivalent to 22 cycles/deg, close to the resolution limit. Stock lenses are available with up to 11.8 grooves $/ \mathrm{mm}$; these could be used for shorter viewing distances or to provide smaller, higher resolution images.

We use a chinrest to keep the observer's head in roughly the correct location. For binocular viewing, we align the image of the projector lamp between the observer's eyes.

This arrangement results in a relatively small image of the projector lamp at the observer, too small for unrestrained viewing and, in any case, much more intense than needed. With our projector, the light intensity was at damaging levels and could not be viewed safely. In order to achieve unrestrained binocular viewing, we placed a high-quality holographic narrow-angle diffuser ${ }^{4}$ against the observer side of the Fresnel lens. The holographic diffuser disperses the lamp light over a small angle (we used $10^{\circ}$ ). The dispersed light has a bivariate Gaussian intensity profile. The diffuser reduced the maximum intensity at the observer to reasonable levels $\left(\sim 10^{5} \mathrm{~cd} / \mathrm{m}^{2}\right)$ by spreading the light over a larger area (about $17 \times$ $17 \mathrm{~cm}$ full width at half maximum) around the observer's eyes. The holographic diffusers used have high transmission efficiency $(\sim 90 \%)$ throughout the visible spectrum. With the lamp image centered between the observer's eyes and spread by the diffuser, intensity at both eyes is equal and does not vary much with small changes in position of the head and eyes.

Image formation. The projected image is formed in the plane of the Fresnel lens (see Figure 3). The projector we used has a minimum focusing distance of $140 \mathrm{~cm}$, farther than desired. At this distance, the image is also too large to fit on the Fresnel lens $(\sim 60 \times 50 \mathrm{~cm})$. In order to focus the image in the plane of the Fresnel lens (about $50 \mathrm{~cm}$ away), we added a large positive achromatic lens 5 immediately in front of the projector's objective lens. This also reduces the size of the projected 


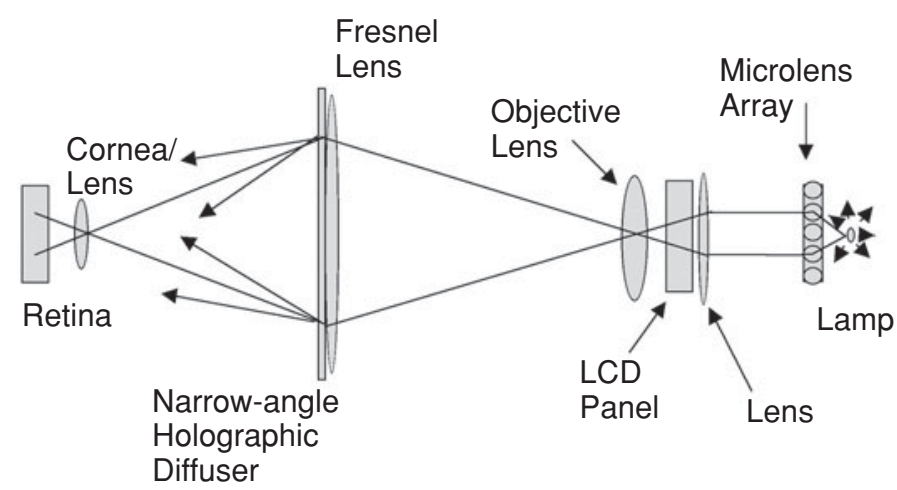

Figure 2. Optical path showing two light rays emanating from a single point within the compact lamp source. Note: not to scale.

LCD image so that it fits on the Fresnel. The built-in optical zoom and focus functions were used to fine-tune the size and focus of the image on the Fresnel lens.

A three-channel display can achieve extremely saturated colors, while still maintaining high light output, using additional dichroic reflecting filters placed inside the projector before or after the LCD or DLP panels. Because of the large amount of light energy that the filters typically block, absorbing filters cannot be used, since they do not survive the heat stress.

To achieve the widest color gamut possible, we used dichroic filters to reduce light that stimulated more than one receptor class at a time. The filters maximize the ratio of S/L cone excitation by the blue channel and maximize the ratio of $\mathrm{L} / \mathrm{M}$ cone excitation by the red channel. ${ }^{6}$ For the green channel, the filter is a compromise that slightly increases the $\mathrm{M} / \mathrm{L}$ cone ratio, while substantially reducing the $\mathrm{S} / \mathrm{M}$ cone ratio. Figure 4 shows the chromatic gamut of the BIGMAX projector after addition of a 430-nm lowpass filter to the blue channel, addition of a 500 - to $550-\mathrm{nm}$ bandpass filter and a $540-\mathrm{nm}$ highpass filter to the green channel, and addition of a 645-nm highpass filter to the red channel. Filtered spectra are shown in Figure 5. The filters reduce display luminance by about a factor of four: Whereas maximum intensity without filters is $\sim 20,000 \mathrm{~cd} / \mathrm{m}^{2}$, the maximum equal-energy white intensity with the filters in place is $\sim 5,000 \mathrm{~cd} / \mathrm{m}^{2}$.

\section{Design Examples}

We present two sample designs: (1) our design (approximately) and (2) a long-throw, low-magnification design that could be used to present stimuli to observers in fMRI experiments. The second design is based only on our experience with the first one; we have not built the second design. In designing our display, we used thin lens equations to make rough guesses about the lenses and distances required, bought several lenses with roughly correct focal length, and then got a working system by trial and error.

First, the projected image needs to be made the desired size and focused at the desired distance. We wanted an image that would fit on the $25 \times 25 \mathrm{~cm}$ Fresnel lens, with the projector as close as possible. The standard

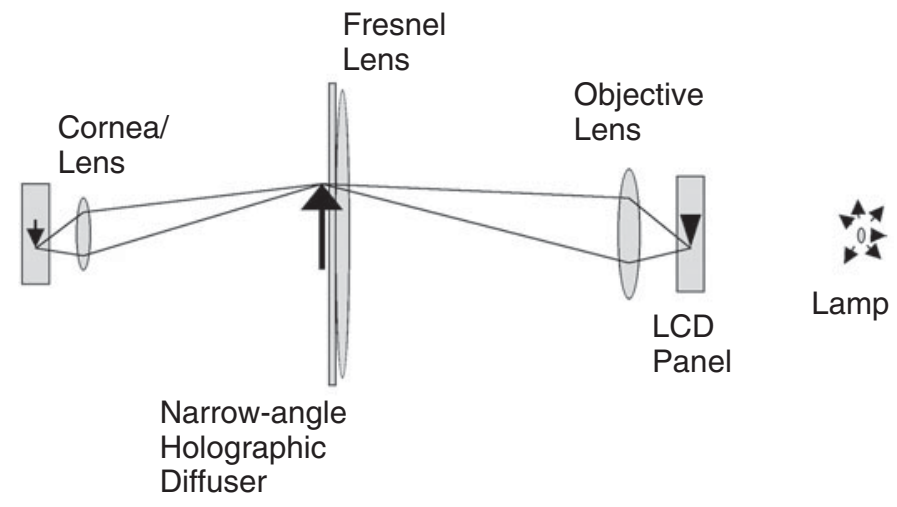

Figure 3. Image formation, showing two rays that pass through a single pixel in the LCD panel after originating from two distinct points in the lamp source. These are separate at the objective lens but are reunited in the image plane at the Fresnel lens and again at the retina. The angular dispersion added by the holographic diffuser is not shown. 

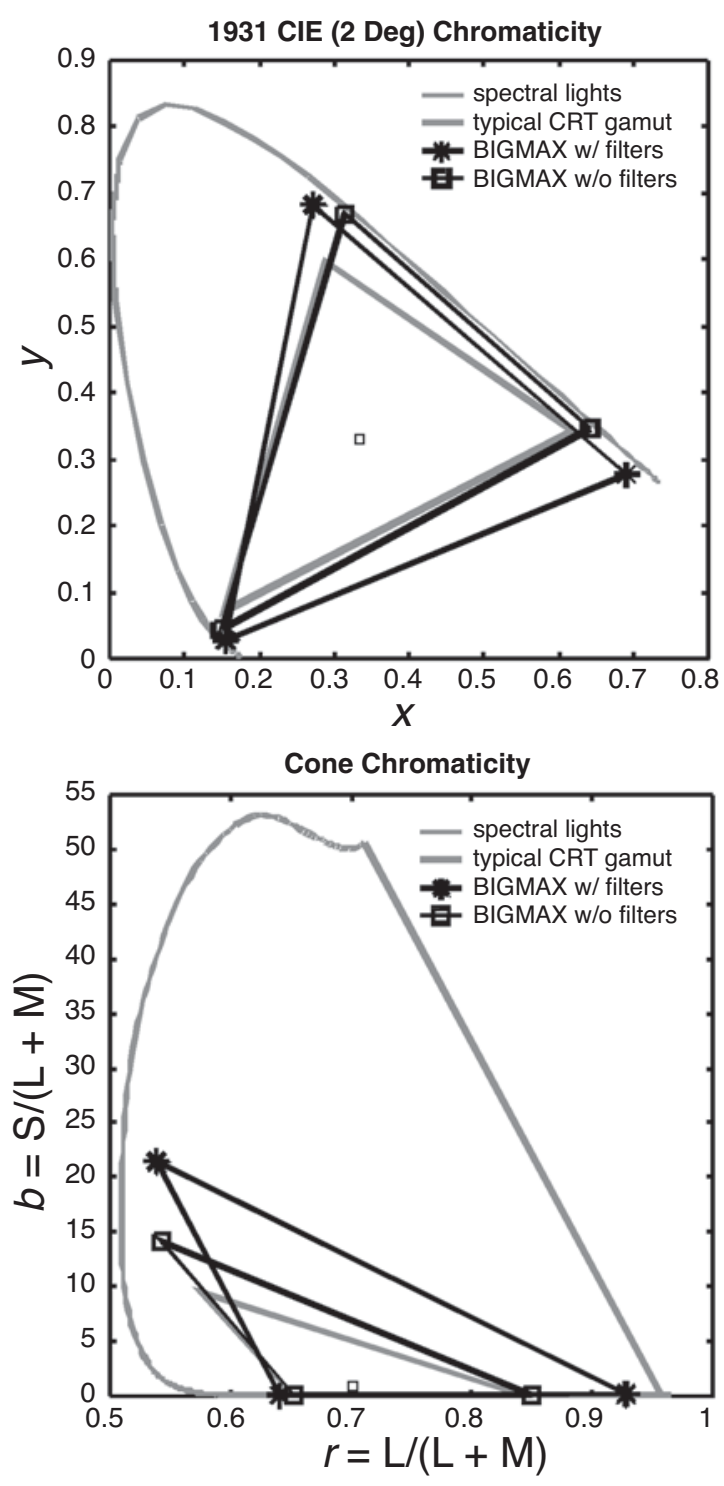

Figure 4. Comparison of device gamuts in 1931 CIE chromaticity (top) and MacLeod-Boynton cone chromaticity (bottom). The BIGMAX display has a wider gamut around equalenergy white (small squares) in both the red-green and the blueyellow directions. The outer line in each diagram is the spectrum locus.

measure of image size is the diagonal screen size. For a standard 4:3 projector image, screen size would have to be

$$
w_{\text {desired }}=\sqrt{(25)^{2}+\left(\frac{3}{4} * 25\right)^{2}}=31 \mathrm{~cm}
$$

for the image to fit on the diffuser. The minimum screen size and distance, using only the standard projector lens, is

and

$$
w_{\min }=79 \mathrm{~cm} \text {, }
$$

$$
d_{\min }=140 \mathrm{~cm}
$$

according to the published specifications. The distance at the desired size, using the standard projector lens, would have to be

$$
d_{\mathrm{d}}=w_{\text {desired }} / w_{\text {min }} * d_{\text {min }}=31 / 79 * 140=55 \mathrm{~cm} .
$$

To achieve this image distance, a positive lens can be added next to the standard projector lens. The focal length would need to be roughly

$$
\begin{aligned}
f_{\text {added }} & =1 /\left(1 / d_{\mathrm{d}}-1 / d_{\text {min }}\right) \\
& =1 /(1 / 55-1 / 140)=91 \mathrm{~cm} .
\end{aligned}
$$

So a $\sim 90-\mathrm{cm}$ lens added in front of the projector should bring the image to a focus on the $25 \times 25 \mathrm{~cm}$ Fresnel lens about $55 \mathrm{~cm}$ from the projector.

Next, the focal length of the Fresnel lens must be chosen to form an image of the projector's light source at the position of the observer. Projector design requires that an image of the light source is formed near the projector lens aperture, so that all the light can exit the projector. This image must be focused at the position of the observer.

For a roughly $15^{\circ}$ diagonal image, the observer must be about

$$
d_{\mathrm{o}}=w_{\text {desired }} / \tan \left(\theta_{\mathrm{d}}\right)=31 / \tan (15)=115 \mathrm{~cm}
$$

away from the display. The required focal length of the Fresnel lens is then approximately

$$
\begin{aligned}
f_{\text {fresnel }} & =1 /\left(1 / d_{\mathrm{d}}+1 / d_{\mathrm{o}}\right) \\
& =1 /(1 / 55+1 / 115)=37 \mathrm{~cm} .
\end{aligned}
$$

Calibration and Performance Measures ${ }^{7}$

Intensity linearization. As with CRTs, the relationship between the digital value sent to the video card and the corresponding analog intensity produced by the display must be characterized, to produce stimuli of known luminance and to make color characterization possible. The input voltage to output intensity transfer function of CRT displays is determined by the physical characteristics of the CRT and can be accurately modeled with a modified power function (Brainard, 1989). The most common characterization procedure is to measure the output intensity for a set of digital input values, fit a modified power function of voltage to the data to smooth and interpolate it, and then generate exhaustive lookup tables from the fit power function, which can be used by the experimental software (or loaded into hardware lookup tables).

However, LCD projector transfer functions typically do not follow a simple power function. We have developed an accurate characterization procedure that can deal with any relatively smooth, monotonically increasing transfer function. The basic steps are as follows.

1. Measure the video card output voltage for every possible digital-to-analog converter (DAC) value.

2. Measure the projector's output intensity for a subset of possible digital values input to the video card. Subtract measurements of the minimum projector intensity (see the explanation below). 


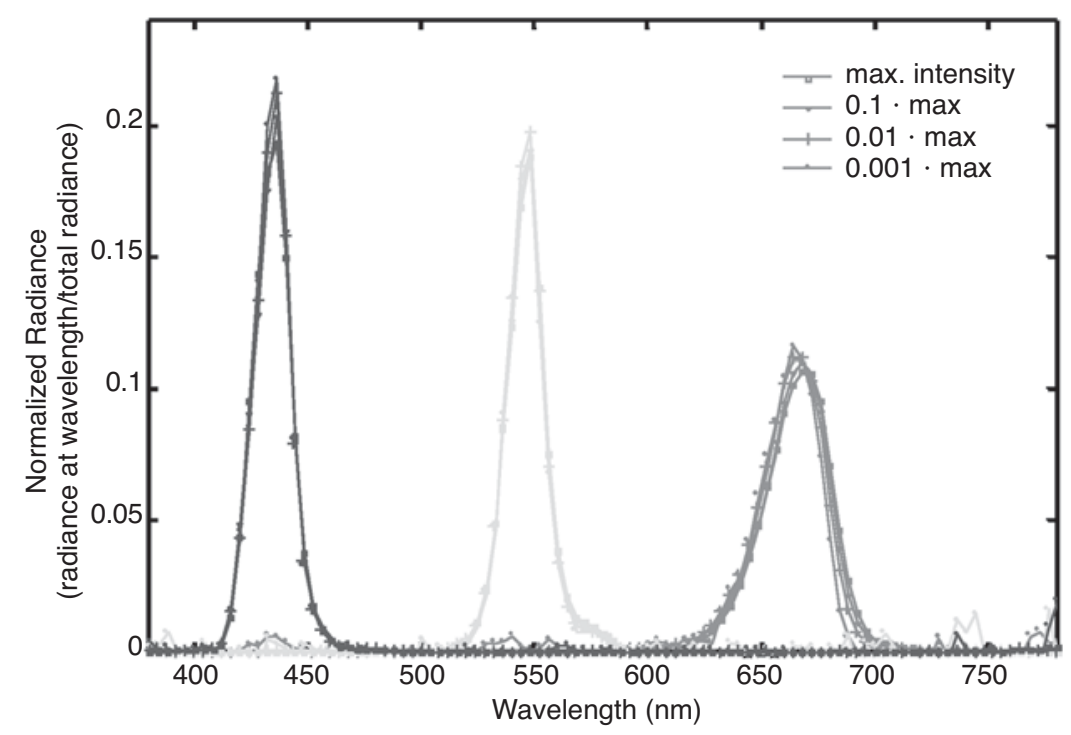

Figure 5. The distribution of radiance over the spectrum is constant with intensity over a 1,000-fold range. Shown are normalized measurements for each color channel at $1,0.1,0.01$, and $0.001 \times$ maximum intensity. For this comparison, the constant off light was subtracted from the measurements, and each resulting radiance distribution was divided by its total radiance.

3. Fit a high-order polynomial (14th or 16 th order seems to work well; lower order polynomials do not follow the data accurately, whereas higher order polynomials oscillate and are affected too much by measurement noise) to the $\log 10$ (voltage) versus $\log 10$ (intensity).

4. Generate a lookup table by using the fit polynomial. Most LCD projectors modulate light from a constantintensity light source by changing the opacity of three separate monochromatic LCD panels (one for each color channel), so one would expect that light from the three channels would add without any interaction. In fact, one would expect more interaction between color channels in CRTs, unless digital signal processing (DSP) mixes video signals for the three color channels. We confirmed that light increments in each of the three color channels add together precisely. This means that three lookup tables, one for each color channel, are enough to precisely characterize the input-output relation of the display.

For reasons that will be discussed below, we decided to subtract the constant off intensity from our intensity measurements, on the assumption that the visual system adapts to or discounts this constant light.

Color characterization. We found that the spectral radiance distribution of each color channel changes very little over a 1,000-fold range of intensities (Figure 5). This makes calibration simple, since it allows desired colors to be generated by mixing the three known spectral distributions. The procedure was as follows.

1. For each color channel, measure the spectral energy emitted by the display with that channel on while the other two channels are off. Also measure the spectral energy with all three channels off.
2. Subtract the off spectrum from the on spectrum for each channel (see the explanation below).

3. Calculate L-, M-, and S-cone excitations for each channel by convolving the measured (incremental) spectra with cone sensitivity functions (cone sensitivities are available on Stockman \& Sharpe's Web site, http://www .cvrl.org).

The $3 \times 3$ matrix of L-, M-, and S-cone excitations for the red, green, and blue channels is used to calculate LMS from RGB, and vice versa.

Contrast ratio and minimum luminance. $L C D$ projectors have limited contrast ratio because they emit some light in the off state. The contrast ratio of our LCD projector display with dichroic filters inserted is 48 (using the ANSI method of measuring a $4 \times 4$ black-and-white checkerboard). However, the early visual system discounts constant background light, so for many vision experiments, the range between the smallest and the largest reliably produced intensity increments is more important than the standard contrast ratio. We measured the ratio of the highest possible intensity increment to the smallest reliably produced intensity increment. The smallest intensity increment that can be reliably distinguished from noise is $1 / 10,000$ of the maximum intensity increment, demonstrating an intensity resolution ample for most vision experiments (Figure 6).

This 4.0 decimal log unit range is equivalent to 13.3 bits, approximately equal to the resolution of the 12-bit video card we used to drive the projector. When one uses the 12-bit card and the lookup tables generated by our calibration procedure, deviations from linearity are small across the range of output intensities: Maximum lumi- 


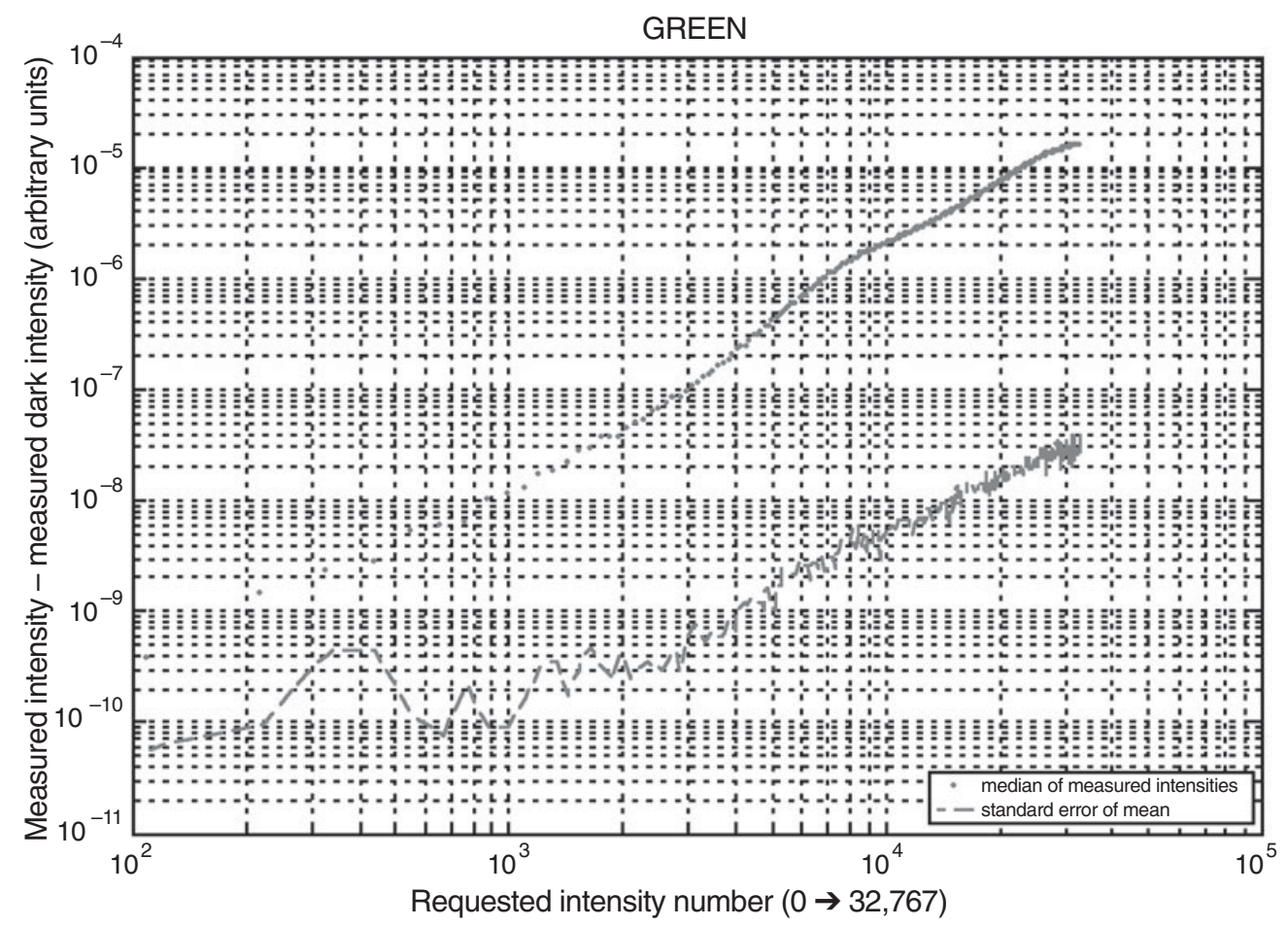

Figure 6. Measured intensity increment versus the requested intensity (DAC) value, green channel.

nance error of a full-intensity stimulus is $1 \%$ and, at the most, $10 \%$ when very small increments of the dark level were called for (see Figure 7).

Spatial characteristics. We found that the spatial resolution of the BIGMAX display is slightly less than the nominal $1,024 \times 768$ projector resolution. This is due partly to light scattered in the optics and partly to the LCD elements, projector electronics, or video card electronics.

To estimate the amount of light scatter in the projector and external optics, we measured the light shining through a small aperture (slightly larger than a single pixel, $500 \mu \mathrm{m}$ ) placed on the observer side of the diffuser. The modulation of light shining through the aperture while surrounding pixels were turned on and off gave a measure of light spread. The on-off modulated surround contained a dark hole centered on the test pixel. Ideally, the amount of light from the test pixel at the center of the dark hole would have been unaffected by illumination of the surround. Figure 8 shows the modulation of light from the test pixel as the surround was switched on and off, as a function of the radius (in pixels) of the dark hole. Modulation is shown as a proportion of the maximum possible on-off modulation. The test pixel was brighter when immediately surrounding pixels were turned on. However, this effect declines rapidly with distance; there is less than $2 \%$ added light from the test pixel when the surround is more than 3 pixels ( 2 arcmin) away.

There is also a modulation of the light intensity of individual pixels that is not due to optics. When the test pixel is turned on at $50 \%$ of maximum intensity (rather than being nominally dark, as in the preceding test), the intensity modulation due to surrounding pixels is greater than that in the previous measurement (Figure 8), becoming less than $2 \%$ only when the surround is more than 24 pixels ( 18 arcmin) away. This indicates that there is more than just scattered light from the surround, since the surround is identical in both cases and would add the same amount of scattered light no matter what the intensity of the test pixels. This pixel interaction may occur during processing of the video signal within the projector.

The errors in intensity are small when stimuli consist of a large number of pixels, since they depend on intensity differences between adjacent or nearly adjacent pixels. However, the errors can be significant when images contain pixel-level detail. We found one experimental situation in which the error was large: single-pixel intensity increments on a large background field. With CRT stimulators, measurements for full-field stimuli are also valid for relatively small test fields surrounded by a different intensity. The lower limit of size is typically on the order of several pixels and is set by video bandwidth (Brainard, Pelli, \& Robson, 2002).

But with LCD stimulators such as the projectors we used, the measured intensity of a single pixel varies strongly as a function of the surround intensity, even when the customary correction is made for the nonlinear voltage-intensity relationship measured for large fields. For the measurements shown in Figure 9, a uniform background field was displayed at an intensity shown on the horizontal axis. A test patch was set to a higher nominal 


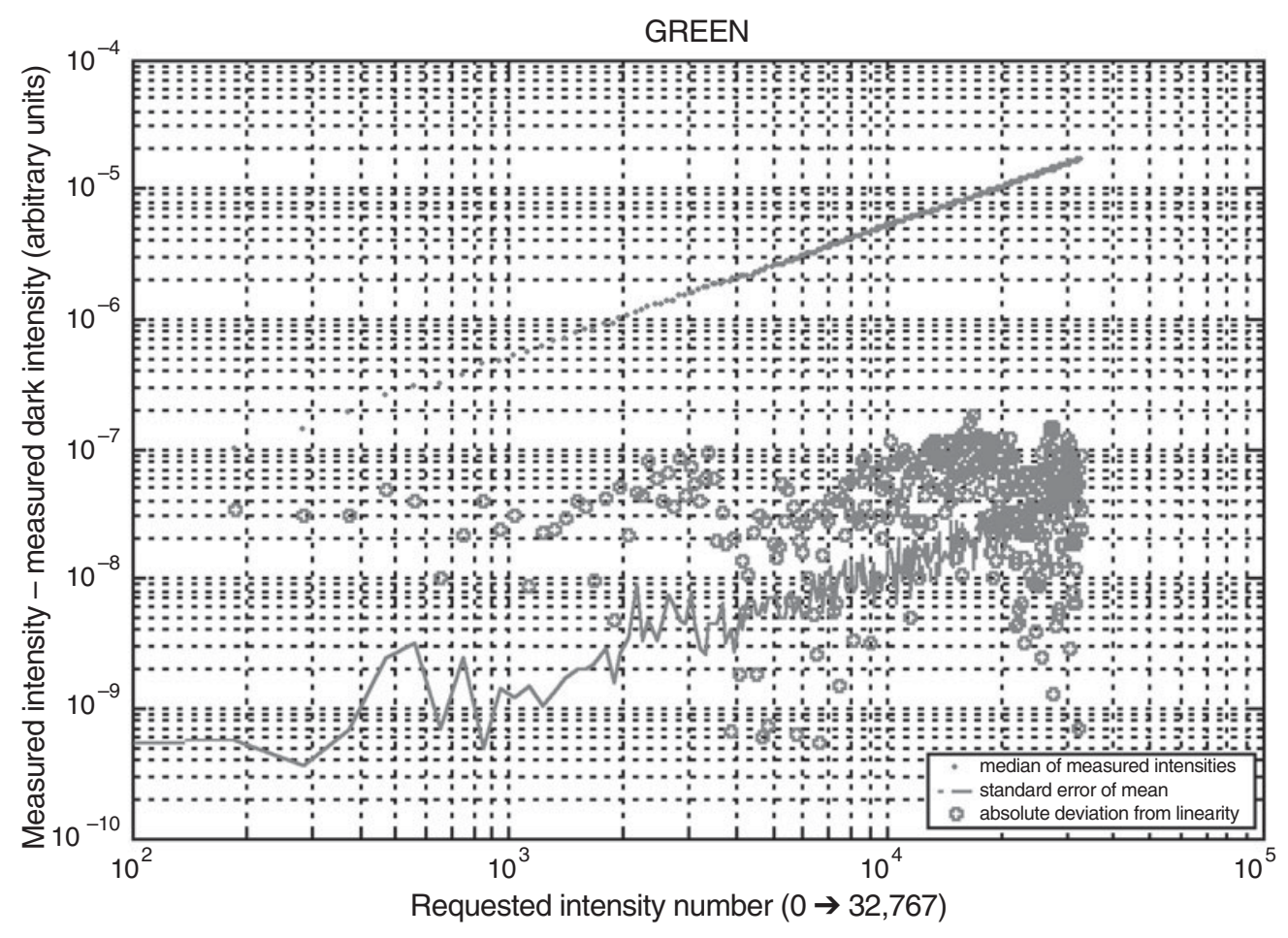

Figure 7. Intensity increment versus LUT-modified DAC value, green channel.

intensity than the background level, and we measured the resulting increase in the actual intensity of the center pixels in that test patch. Pixels in small test patches were found to fall short of the values obtained for large fields by up to a factor of seven. The shortfall was greater with small increments on dark backgrounds, especially backgrounds of less than $5 \%$ of maximum intensity. Although inconvenient, these errors appear to be consistent, so patches of just a few pixels can be accurately rendered by making careful calibrations, using the particular stimulus configuration of interest. If these pixel-to-pixel dependencies are due to interactions within the LCD panels, rather than to video signal processing, use of a threechannel digital micromirror projector could eliminate this problem.

Luminance and color uniformity. There are measurable nonuniformities in luminance and chromaticity across the display screen. Table 1 shows measurements for a high-intensity, near-white field. These are constant and probably not important in many applications, since they are not easily visible (CRT displays also have significant luminance nonuniformities). For critical applications, it may be possible to correct these nonuniformities in software or by using a negative photographic mask.

\section{Safety}

Light intensity produced by this display will reach damaging levels if the display is operated without the diffuser, since all the projector light will then be focused in a very small region. Light intensity can also easily reach damaging levels if the display is operated without sufficiently dense color or neutral density filters. Personnel should never place their eyes in the light path and look in the direction of the projector; this is especially easy to forget during setup of the display. Experimental subjects should not view the display until the safety of the light level at the location of the subject is verified to be safe and until the display is approved by the institution's review board for the protection of human subjects. Consult standard texts (Optical Society of America, 1995; Sliney \& Wolbarsht, 1980) to ensure that infrared, visible, and ultraviolet light delivered to subjects' eyes is at safe levels.

\section{Summary}

We present a modified Maxwellian view display that produces very high intensity images, even with unrestrained binocular viewing. The display is capable of producing highly saturated colors, approaching the sat-

Table 1

Luminance and 1931 CIE $\left(2^{\circ}\right)$ Chromaticity at Nine Locations on the Display Screen for a Uniform Near-White Field

\begin{tabular}{ccc}
\hline $4,800 \mathrm{~cd} / \mathrm{m}^{2}$ & $5,000 \mathrm{~cd} / \mathrm{m}^{2}$ & $4,700 \mathrm{~cd} / \mathrm{m}^{2}$ \\
$x=0.37, y=0.41$ & $x=0.37, y=0.41$ & $x=0.37, y=0.40$ \\
$5,400 \mathrm{~cd} / \mathrm{m}^{2}$ & $5,500 \mathrm{~cd} / \mathrm{m}^{2}$ & $5,000 \mathrm{~cd} / \mathrm{m}^{2}$ \\
$x=0.35, y=0.40$ & $x=0.35, y=0.38$ & $x=0.36, y=0.38$ \\
$5,300 \mathrm{~cd} / \mathrm{m}^{2}$ & $5,400 \mathrm{~cd} / \mathrm{m}^{2}$ & $4,500 \mathrm{~cd} / \mathrm{m}^{2}$ \\
$x=0.34, y=0.38$ & $x=0.34, y=0.36$ & $x=0.35, y=0.36$ \\
\hline
\end{tabular}




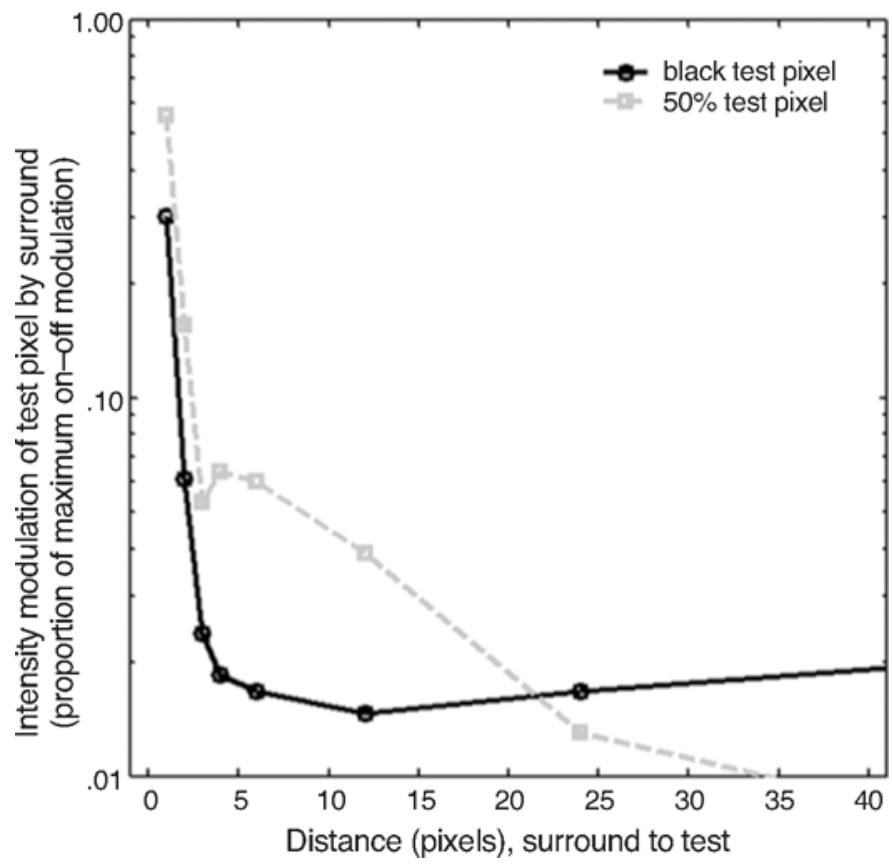

Figure 8. Modulation of light shining through a 500- $\mu \mathrm{m}$ aperture when surrounding pixels are turned fully on and off. Modulation is shown as a proportion of the maximum possible modulation (test pixel on-test pixel off).

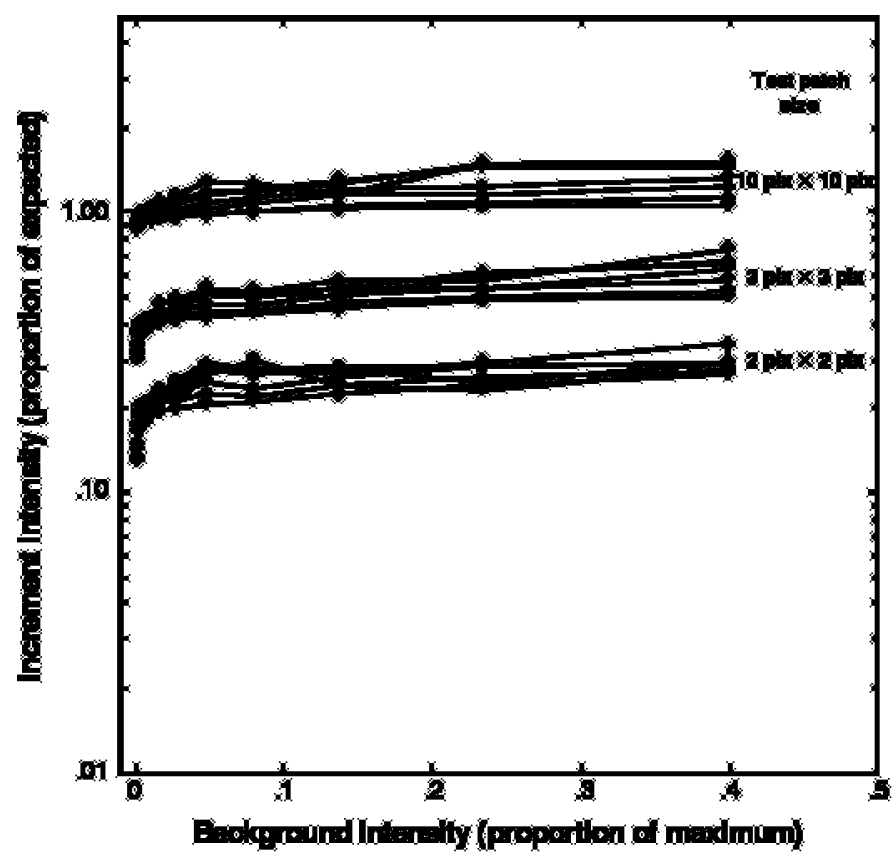

Figure 9. Shortfall of increments in intensity of small patches when the background intensity varies. Intensity measurements of the center pixels of each test patch were made through a 500- $\mu \mathrm{m}(\sim 2 \times 2$ pixel $)$ aperture. Each line represents the measured increment intensity as a proportion of the requested increment, and ideally, each line would be horizontal at 1.0. With dark backgrounds of less than $5 \%$ of maximum intensity, the incremental intensity of small test patches is reduced by as much as a factor of seven below the value expected on the basis of calibration with large stimuli. 
uration of spectral colors. It has excellent spatial and good temporal resolution and can be calibrated for demanding applications, using the procedures we have described.

Many applications are possible, including experiments requiring very high light levels, natural light levels, or extremely saturated colors. The display is not expensive, requires only a moderate amount of work to set up, and can be customized for desired viewing distance and angular subtense. A compact display could be constructed for in-office clinical diagnosis.

We have used the display to make sensitive, objective measurements of the optical quality of the eye after lens replacement surgery. A patient's complaints of glare and halos were objectively corroborated, and measurement could be compared with standard estimates of the point spread function (Beer, MacLeod, \& Miller, 2003). We also used the display for research in which color coding in the human visual system was investigated (Beer \& MacLeod, 2003). These experiments required high color saturation and would not have been possible without the extended gamut of this display.

The Extended Maxwellian View (BIGMAX) is a lowcost display system with high intensity, resolution, and saturated colors for use in clinical, applied, and pure vision research.

\section{REFERENCES}

Beer, D., \& MacLeod, D. I. A. (2003). Color selectivity in metacontrast: Asymmetrical and anisotropic. Journal of Vision, 3, 138a.

Beer, D., MacLeod, D. I. A., \& Miller, T. (2003). The black hole test for retinal light spread. ARVO Meeting Abstracts, 44, 4194.
BrainaRd, D. H. (1989). Calibration of a computer controlled color monitor. Color Research \& Application, 14, 23-34.

Brainard, D. H., Pelli, D. G., \& Robson, T. (2002). Display characterization. In J. Hornak (Ed.), The encyclopedia of imaging science and technology (pp. 172-188). New York: Wiley.

Optical Society of America (1995). Handbook of optics (2nd ed.). New York: McGraw-Hill.

Rushton, W. A. H., \& Henry, G. H. (1968). Bleaching and regeneration of cone pigments in man. Vision Research, 8, 617-631.

Sliney, D., \& Wolbarsht, M. (1980). Safety with lasers and other optical sources. New York: Plenum.

Stockman, A., \& Sharpe, L. Colour and vision database. Available at www.cvrl.org.

Westheimer, G. (1966). The Maxwellian view. Vision Research, 6, 669-682.

\section{NOTES}

1. It should be possible to use other projector technologies - for example, Texas Instruments' DLP_-but we have not tried them.

2. InFocus Corporation, 27700B SW Parkway Avenue, Wilsonville, OR 97070-9215 (www.infocus.com), $\$ 3,000$. This projector is a repackaged version of the Sanyo PLC-XP21N.

3. Fresnel Technologies Inc., 101 West Morningside Drive, Fort Worth, TX 76110 (www.fresneltech.com). Aspheric Fresnel Lens, \$45 each.

4. Physical Optics Corporation, 20600 Gramercy Place, Suite 103, Torrance, CA 90501 (www.poc.com). Light Shaping Diffusers, about $\$ 300$.

5. One source for these are suppliers of telescope objectives-for example, Island Eyepiece, Box 133, Mill Bay, BC, V0R 2P0 Canada (www.islandeyepiece.com). We bought a Vixen Fraunhofer objective lens (about \$200).

6. LINOS Photonics Inc., 459 Fortune Blvd., Milford, MA 01757 (www.linos.com). Dichrolight Filters B-42 Dark Blue, G-50/55 Primary Green, Y-54 Amber, R-65 Primary Red, each about \$35.

7. A set of MATLAB scripts implementing the calibration described above is available on Beer's Web site at http://psy.ucsd.edu/ rdbeer.

(Manuscript received January 8, 2004; revision accepted for publication August 14, 2004.) 